\title{
Mud Pulse Signal Extraction and Noise Cancellation based on Adaptive LMS and NLMS Algorithms Performance
}

\author{
Saleh M. Mwachaka \\ National Demonstration Center for Experimental Electrical \& Electronical Education, \\ Yangtze University, Jingzhou, Hubei 434023, China.
}

\begin{abstract}
During logging-while drilling data collection and transmission, strong noise and interferential signals contaminate mud pulse encoded signals. Varying and unpredictable statistical noise characteristics severely influence logging mud pulse signal measurement precision and reliability; making the raw signal extraction from the received signal becoming the key problem. Adaptive filter algorithms perform statistical estimation of the unknown signal to eliminate noise signals and overcome the problem of fixed coefficient digital filters. This paper researches the adaptive noise cancellation method to realize filter performances based on field mud pulse signal submerged into noise signal. MATLAB simulations are used to implement and simulate adaptive filter least mean square (LMS) and normalized least mean square (NLMS) algorithms. Well-site field mud pulse signal characteristics simulation results are used to compare and analyse the filter algorithm performance capability. Simulations show that the mud pulse input signal contains the direct current (DC) offset frequency and changes the sign level and channel characteristics. Based on MATLAB simulation results analysis, mud pulse useful signal is extracted, direct current offset and noise frequency components suppressed. Experimental results of the proposed method demonstrate the feasibility to apply the solution into mud pulse logging-while drilling systems. The research has presented a systematic step-by-step noise cancellation method to overcome the undisclosed implemented steps or signal spectral characteris tics of different existing drilling signal models and simulation environments.
\end{abstract}

Keywords: Mud Pulse System; Signal Processing; Adaptive Noise Cancellation; LMS; NLMS.

\subsection{INTRODUCTION}

Logging-while drilling (LWD) signals experience pressure fluctuations and uncertainty that potentially change the signal properties throughout data transmission.During data transmission, mud pulsesystem modulates drilling mud circulating along the borehole to create pressure pulses, [1][4]. Downhole pressure pulsescarrying drilling information are generated, encoded and transmittedto the surface receiver system for detection, processing and decoding. However, due to the complex downhole conditions, unpredictable and complex adjustable signal impairmentsseverely influence the signal transmission process which impacts the surface received signal quality and precision. The transmission medium is susceptible to signal noises from mud pump, drilling vibration, signal attenuation, reflection signals and other random noises that flood the original pulse signal, [5][7]. Mud pump signals and reflection frequency components account for more than $80 \%$ of the received signal noises and their frequency distribution may coincide with transmitted mud pulse signal, [8]. Noise frequency components may have higher amplitudes or frequencies than that of the encoded signal, hence, weakening the signal detection and reception, [9]. These noise signals complicate the surface signal detection, extraction, decoding and interpretation processes. Prior to signal decoding, surface received signals require complex signal detection and must be filtered first to get rid of the unwanted frequency components so that correct downhole information can be recognized, [9]. Due to spectrum aliasing of noise signals and mud pulse signal, conventional signal processing methods cannot effectively suppress these noise signals. Mud pulse systems are required to deploy robust signal processing techniques to adapt the harsh working environment that adversely impact the effective downhole drilling signals.

Numerous surface signal processing techniques such as signal decoding, noise cancellation and channel equalization have enhanced mud pulse drilling performances in tests and field applications.Signal processing techniques based on Fourier transform, Wavelet transform and adaptive filter method are the currently applied noise cancellation methods. Fourier transform method uses two pressure transducer measurements added with active pump sensor signal to compute the signal transfer function which estimates the downhole telemetry signal, [10]-[13]. However, Fourier transform de-noising method is suitable for stationary signals and cannot effectively distinguished the high noise frequency changes over time of non-stationary signal effects, [14], [15]. Additionally, this technique is complex as it is difficult to estimate accurately the phase difference required quantifying the time delay between the pump sensor and pressure sensor signals. Wavelet transform de-noising technique was developed to overcome the Fourier transform coefficients (sine or cosine functions) undulating characteristics to infinity, [16], [17]. Wavelet transform divides the signal into space and scale (time and frequency) without losing any useful information of the original signal which ensures useful information extraction from the noised signal, [17]. The study [18] and [19] implemented the application of wavelet transform technique to detect and extract effective signal from the received non-stationary continuous mud pulses. However, the selection of the wavelet base function and scale range is complex and lacks the general method. 
Adaptive filtering has been used widely in a range of digital signal processing applications, including echo cancellation, channel equalization, noise cancellation, and beam forming. Logging-while drilling (LWD) researchers have studied the application of adaptive filter algorithms into noise cancellation. The method ability to adapt unknown noise statistics and time varying signal characteristics has shown optimal noise cancellation filtering and attracted scientific interests. The study [20] applied the LMS algorithm to adaptively track harmonic pump noise signals to eliminate pump noise frequencies and reconstruct mud pulse signals; [21] studied adaptive noise cancellation method to extract weak electromagnetic LWD signals from large amounts of well-site noise using modified LMS algorithm. These literatures provide an imperative novel noise cancellation application into logging mud pulse data transmission. Though the method is complex and not straightforward to implement, experimental and simulated results show that adaptive LMS algorithm generate quite good noise reduction results after certain successive iterations.

However, most of the existing literatures discuss the adaptive noise cancellation and signal extraction techniques without evidently disclosing the applied method steps or the signal spectral characteristics. Other studies use computer simulations to generate drilling signal added with random noise signals. Mud pulse signal extraction based on mathematical theory analysis and numerical simulation have been implemented by, [19], [22], [23]. Other studies have used laboratory environment to build and simulate the actual drilling environment where short transmission distance were implemented. The study [24] initially simulated the mud signal extraction method and later, in order to verify the implementation, the hydraulic circulation laboratory was built to simulate the actual drilling environment. The study [12] simulated adaptive noise cancelation to extract the information signal from pump noise added with random noise interferences and later field experiments were implemented on $3 \mathrm{~km}$ drilling operation. The research [6] applied FIR filter algorithm to cancel pump impulse noise on a target experimental mine of $3.5 \mathrm{~km}$ deep and the mud viscosity of $10 \mathrm{mPa}$.s. Other studies evaluate and compare LMS algorithms using sinusoidal signals submerged into a zeromean noise implementing Gaussian input signal distribution. These assumptions showed good performances and robustness of the implemented methods. However, the different drilling signal simulation environments and implementation models contribute to inconsistency conclusions and even compromise between the computational burden and accuracy.

In this study, the adaptive LMS and NLMS filtering algorithms are implemented to adaptively estimate and extract effective mud pulse signal submerged into large amount of well-site noise signals. The main objective of this research is to examine the performance of LMS and NLMS signal processing algorithms based on adaptive filter noise cancellation. The research demonstrates the positive mud pulse signal noise cancellation application using adaptive filter algorithms. MATLAB simulation results to verify the viability of the proposed method are presented.Step by step of the implemented methodology, hypotheses and the experimental simulations are clearly explained. Extracted signal analysis in frequency spectrum is discussed to further substantiate the effectiveness of the noise cancellation method. Positive mud pulse noise contaminated signal spectra characteristics and experimental simulation results of the adaptive noise cancellation method are presented in section 3 and 4 respectively.

\subsection{ADAPTIVE FILTER THEORIES}

Unpredictable and time varying noise signals severely influence mud pulse system precision and reliability during drilling data collection and transmission. Limitations of noise signals in digital signal processing have gained attention into mud pulse data transmission systems. In order to extract effective positive mud pulse signal submerged into noise frequencies from well-site field data, adaptive filter was used. Adaptive filter algorithms have been widely employed into noise cancellation techniques. Adaptive filters have the ability to perform statistical estimation of unpredictable noise or time-varying unknown signals where fixed coefficient FIR and IIR filter cannot achieve optimal filtering, [25].The filter automatically adjusts its initial parameters to change their performance without prior knowledge of signal and noise characteristics. The principal advantage of the method is in its adaptive capability, its low output noise, and its low signal distortion, [26]. Based on in-depth study of adaptive filters, this study uses the linear phase finite impulse response (FIR) to achieve optimal filtering. Linear phase filters are suitable as they ensure right parameters to be carried out from the filtered signal; non-linear adaptive filters have more complex calculations, [27].

The method has a self-modifying frequency response ability to cancel noise frequency component which overlaps with unrelated signal in the same frequency range, [28]. Nevertheless, adaptive FIR filters have strict linear phase characteristic and good stability, making them convenient for programming and real-time signal processing applications, [6]. Adaptive filter automatically adjust the filter parameters of the present moment using the parameters of a moment ago to adapt the random signal statistical changes. The method updates the filter weights to converge to the optimum filter weight by finding the gradient of the mean-square error (MSE), [29], [30]. The filter weight estimates are required to minimize the signal error, $e(n)$, between the output signal, $y(n)$, and the desired signal, $d(n)$.

During this work, adaptive LMS and NLMS algorithms are applied to study noise cancellation performances on received well-site positive mud pulse signal characteristics. MATLAB simulations are used to realize random signal statistical properties based on modified typical adaptive noise cancellation (ANC) method shown on Figure 1. 


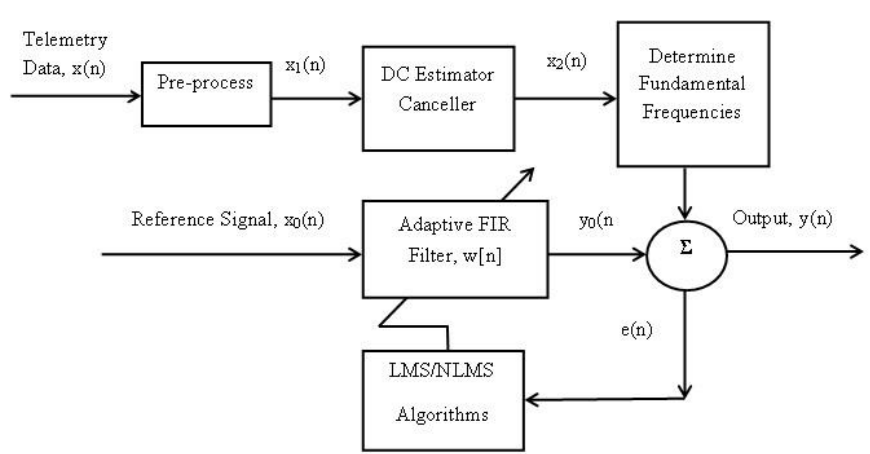

Figure 1: Modified Adaptive Noise Cancellation.

Discrete mud pulse input signal, $x(n)$, is pre-processed to generate MATLAB accessible data, $x_{l}(n)$. The DC offset component in $x_{1}(n)$ data is removed to get $\mathrm{x}_{2}(\mathrm{n})$ signal. Studied $x_{2}(n)$ fundamental frequencies are adaptively processed to produce the output signal, $y(n)$, by a convolution with filter's weights, $w(n)$. The filter output is, $y(n)$, is subtracted from desired signal, $d(n)$, to obtain an estimation signal error, $e(n)$. For each iteration, the error signal is minimized by adjusting the filter's weights for the next time instant. The reference input signal provides the correlated version of the sinusoidal interference. The next subsection 2.1 and 2.2 presents theoretical derivatives of the adaptive LMS and NLMS algorithms noise cancellation method.

\subsection{LMS Algorithm}

LMS algorithm is a stochastic gradient-based algorithms as it utilizes the gradient vector of the filter tap weights to converge towards the optimal Wiener-Hopf equation, [31]. LMS algorithm adjusts and modify the adaptive filter taps to calculate the adaptive filter coefficients by an amount proportional to the instantaneous error estimates of the gradient, [32]. The algorithm makes successive corrections of weight vector in the negative gradient direction that finally concludes to minimize the mean square error (MSE) by iterative method. The algorithm neither requires correlation function calculation nor matrix inversions, [33]. The output signal vector, $y[n]$, is the convolution function between the input signal vector, $\mathrm{x}[\mathrm{n}]$, and the filter's weight vector, $w[k]$, expressed as:

$$
y(n)=\sum_{n=0}^{m-1} w(n) * x(n-k)
$$

The difference between the desired signal, $d(n)$, and the filter output, $y(n)$, is given by:

$$
e(n)=d(n)-y(n)
$$

The LMS algorithm weight vector, $w(n)$, is updated to get the next weight value vector, $w(n+1)$, using equation 3 .

$$
w(n+1)=w(n)+\mu * e(n) * x(n)
$$

The LMS algorithm step size parameter, $\mu$, is a positive constant that determine the filter convergence speed and overall algorithm behavior, as shown in equation 3. By repeating updating, $w(n+1)$, the mean square error (MSE), $E\left[e^{2}(n)\right]$, is minimized.

\subsection{NLMS Algorithm}

LMS algorithm is limited to its sensitivity to input signal, $x(n)$, and scaling factor; creating difficulties when choosing step size, $\mu$, that guarantees system stability, [34]. NLMS is a variant of the LMS algorithm that solves this problem by normalizing the input signal power. NLMS is a stable adaptive filter controlled by the step size parameter that manages the filter convergence rates, tracking ability speed and amount of steady-state mean-square error (MSE), [35].The algorithm realizes the unknown signal varying statistical properties by calculating the appropriate filter step size based on the instantaneous energy of the input signal.Classical NLMS algorithm updates the weights as follows, [36]:

$$
e(n)=d(n)-y(n)=d(n)-w^{H}(n) * x(n)
$$

Where:

$\mathrm{H}:$ Hermitian= Conjugate Response.

$$
\begin{aligned}
& \mu(n)=\frac{\alpha}{x^{T}(n) * x(n)} \\
& w(n+1)=w(n)+2 * \mu(n) * e(n) * x(n)
\end{aligned}
$$

Substituting equation 5 into equation 6 , the new equation changes to:

$$
w(n+1)=w(n)+\frac{2 * \alpha}{x^{T}(n) * x(n)} * e(n) * x(n)
$$

The recurrence formula, equation $7, \alpha$ is a constant ranging, $0<\alpha<2$. In order to avoid the denominator to be equals to 0 , a small positive constant, $0<\gamma<2$, is added. Therefore, the updating iteration formula of NLMS algorithm becomes:

$$
w(n+1)=w(n)+\frac{2 * \alpha}{\gamma+x^{T}(n) * x(n)} * e(n) * x(n)
$$

The derived equation 8 shows that, NLMS algorithm has a time-varying step size, $\mu(n)$ which differentiates NLMS from the standard LMS algorithm. The condition $0<\alpha<2$ makes the NLMS algorithm to give better convergence characteristics than the LMS algorithm at a price of greater residual error, [37]. NLMS algorithm derived equation divides the step size parameter to the input signal vector power which reduces the impact of noise signal.

\subsection{EXPERIMENTAL METHODOLOGY}

Labview VI File Viewer Pallete was used to plot the surface received .tdms channel data into time domain so that the signal characteristics can be examined. MATLAB function convertTDMS() was utilized to import large TDMS data file into matlab data format, .mat, for further time and frequency domains signal processing analysis. Figure 2 presents the time series of the positive mud pulse channel data plotted in MATLAB which revealed same Labview VI data plot. During the signal analysis, the first 360,118 discrete samples were used to study the signal characteristics. Fast Fourier transform (FFT) MATLAB function was used to present the data in frequency spectrum, Figure 3. Based on mud pulse spectral analysis, the received signal can be roughly divided 
into three parts; strong direct current (DC) frequency, weak low-frequency $(0.5$ to $1.5 \mathrm{~Hz})$ and high-frequency noise.

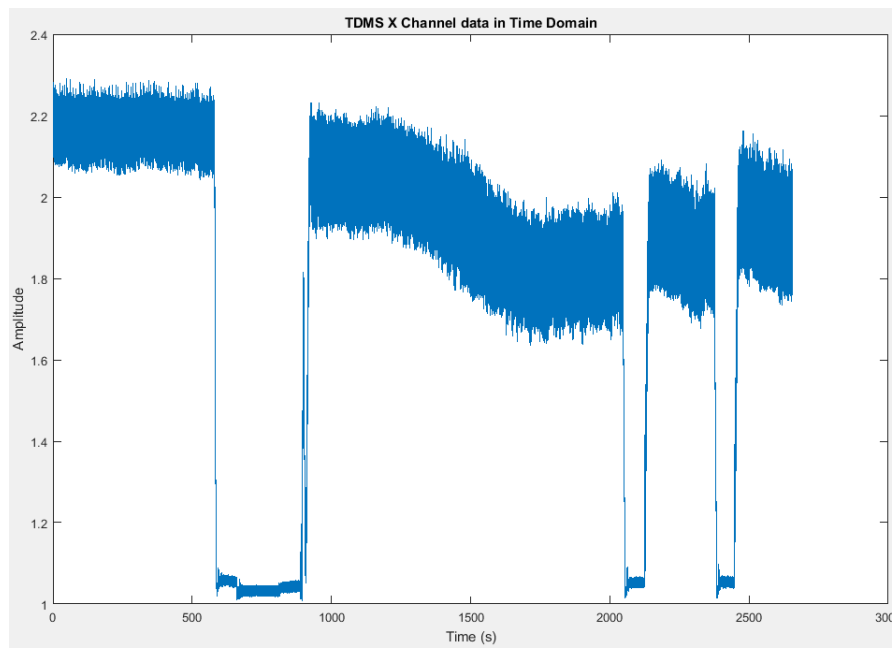

Figure 2: Mud Pulse Channel Data- Time Domain

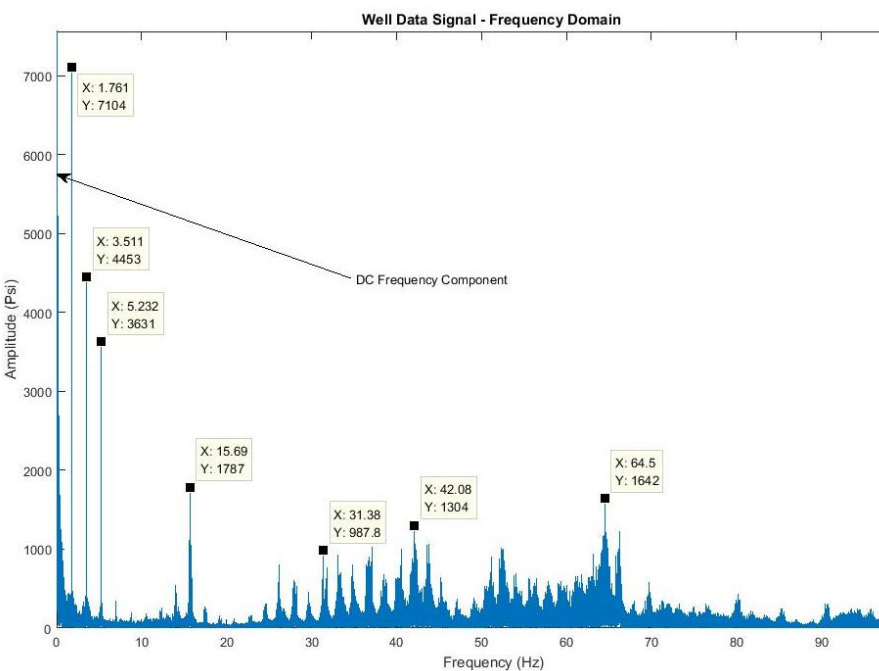

Figure 3: Mud Pulse Data Signal- Frequency Spectrum Analysis.

Direct current frequency offsets must be initially suppressed before applying the noise signal cancellations using adaptive filter algorithms. DC offsets present a significant residual error on the state and error signals causing excess MSE at steady state, [38], [39]. This effect limits the performance of the LMS and NLMS algorithms to realize noise cancellation applications. The application of the adaptive dc tap in combination with a digital LMS algorithms can be used to eliminate the excess MSE due to dc offsets, [40]. However, the implementations of the dc tap using unknown mud pulse signal characteristics will be complex in this case and any performance improvement obtained would be irrelevant. MATALAB object DCBlocker was applied to perform the dc blocking filter operations. The method uses FIR DCBlocker object to remove the DC offset by blocking the DC component from each channel column vector of the input signal, $x[n]$. A step () method was used to pass the created DCBlocker object and input signal samples to generate the DC removed filter output frequency response.
MATLAB simulation environment was used to implement adaptive LMS and NLMS algorithms noise cancellation filtering performances. Experiments are performed on surface received positive mud pulse signal containing unknown noise characteristics without DC offset frequency to avoid any varying signal variations. LMS algorithm variants are evaluated based on filter convergence performances throughout noise cancellation application. The adaptive filter's transfer function must converge to, or near to, the unknown system's transfer function after certain number of iterations. The magnitude difference between the two transfer functions directly relates to the filter error signal. The error signal is used to determine filter convergence, which is a nonzero value for non-ideal applications. MATLAB linear phase constrained low-pass FIR filter method, fir1(k,mu), was used to identify the unknown filter and correlate the noise signal. The LMS variants filter object, dsp.LMSFilter, performs a number of iterations to estimate the filter weights required to minimize the error between the output signal and the desired signal. The technique solved the Wiener-Hopf equation to find filter coefficients while attaining filter optimal solution. Using the same filter design parameters and same set dsp.LMSFilter object methods, simulation results are analyzed based on the implemented LMS and NLMS convergence performances with similar conformity.

Before simulating the individual adaptive filter algorithm, design parameters, filter length and step-size, are necessarily set-up first to get best filter coefficient estimates. These parameters affect the filter convergence rate and specify how accurately the algorithm modeled the noise cancellation application. Choosing the best choice filter length and stepsize values is a complex and trial-and-error process which require numerous simulation attempts. The filter lengths affect the system convergence rate, computational resource requirements, minimum MSE and steady state error of the resulting system; while, the filter step-size values determine the amount of corrections taken to adapt from one iteration to the other. Selection of a suitable step size value is imperative to the algorithm performance. If the value is relatively too small the time the adaptive filter takes to converge on the optimal solution will be too long; if is too large the adaptive filter may become unstable and its output diverges, never reaching convergence, [21], [30], [41]. These literatures reveal that, smaller step-size value improves filter convergence accuracy at the expense of the time it takes to adapt the signal characteristics of the unknown noise signal.

During this study, numerous subjective filter length and stepsize values were used to simulate the adaptive filter LMS and NLMS algorithms noise cancellation evaluations. Presented results applied adaptive filter lengths 16, 32, 64 and step-size values $0.02,0.05$ and 0.1 to simulate the individual algorithm performance. These initial filter designed values were found to provide distinctive results for both algorithms. The filter performances were compared based on filter MSE-gradient, filter convergence rate, steady state error and noise reduction ability. In order to achieve good results, individual step-size value simulated each of the three filter lengths impact on the modeled algorithm. 


\subsection{SIMULATION RESULTS AND DISCUSSION}

Simulation results to demonstrate the DC offset frequency component decay and the adaptive FIR filter LMS and NLMS algorithms noise cancellation performances are presented.

\subsection{Decaying DC Offsets}

The mud pulse spectral analysis in Figure 3 shows that, the signal contains high amplitude frequency component at zero frequency $(0 \mathrm{~Hz})$. The signal presents the direct current (DC) noise frequency whose amplitude is unknown. Simulation result presented in Figure 4, shows that the DC frequency component is evidently decayed to avoid any undesirable signal characteristics during the mud signal extraction process. The performance of the proposed DC offset method can be implemented and used for phasor estimation applications.

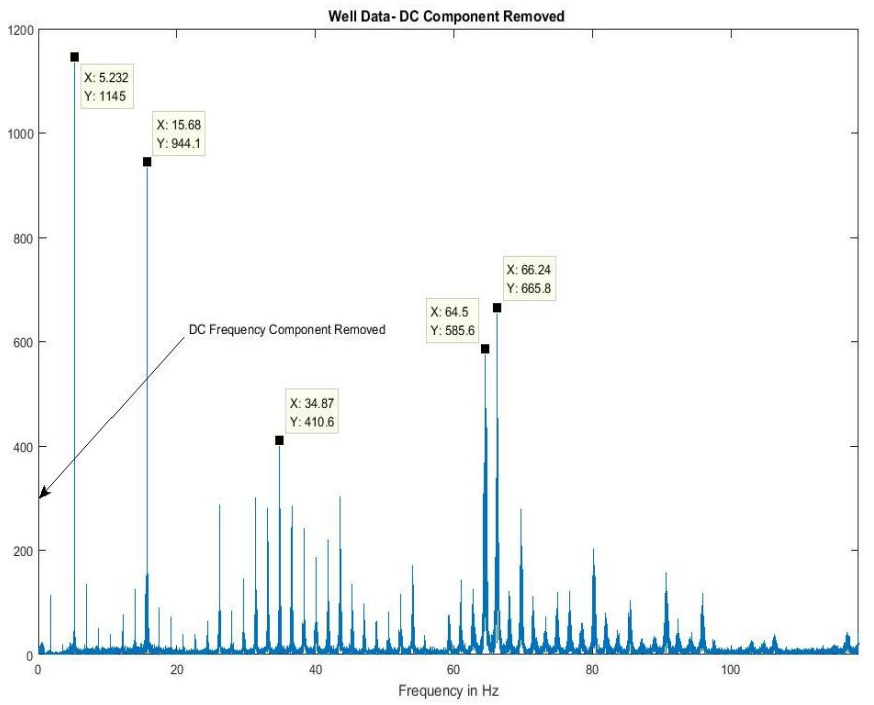

Figure 4: Mud Pulse Data - DC offset Frequency Removed.

\subsection{LMS and NLMS Algorithm Performances}

Table 1 summarize the estimated MSE-gradient values for both adaptive LMS and NLMS algorithms based on individual simulated step sizes and filter lengths.

Table 1: Estimated Mean Square Error (MSE)-Gradient Summary.

\begin{tabular}{|l|l|l|l|}
\hline $\begin{array}{l}\text { Step-Size } \\
\text { Value }\end{array}$ & $\begin{array}{l}\text { Filter Length } \\
\text { Value }\end{array}$ & $\begin{array}{l}\text { LMS } \\
\text { Gradient }\end{array}$ & $\begin{array}{l}\text { NLMS } \\
\text { Gradient }\end{array}$ \\
\hline \multirow{4}{*}{$\mu=0.1$} & 16 & 0.07044 & -0.2142 \\
\cline { 2 - 4 } & 32 & 0.03576 & -0.03359 \\
\cline { 2 - 5 } & 64 & -.0 .01967 & -.0 .06125 \\
\hline \multirow{3}{*}{$\mu=0.05$} & 16 & 0.07439 & -0.07749 \\
\cline { 2 - 5 } & 32 & 0.0383 & -0.02459 \\
\cline { 2 - 5 } & 64 & -0.01448 & -0.0441 \\
\hline \multirow{3}{*}{$\mu=0.02$} & 16 & 0.0783 & 0.006173 \\
\cline { 2 - 5 } & 32 & 0.04093 & 0.006458 \\
\cline { 2 - 5 } & 64 & 0.000060856 & -0.02531 \\
\hline
\end{tabular}

Based on algorithms result presented in Table 1 combining with approaches applied by Hwang and $\mathrm{Li}$ [42], the step-size controls the mean-square error (MSE) of the weightedaverage gradient vector. The step size is updated based solely on the input mud pulse signal to achieve the optimal noise cancellation objective. In this approach, each adaptive algorithm is updated based on the function of the error signal. The interpretation of the signal error, $e(n)$, response vary depending on the impact of adaptive filtering parameters on MSE-gradient sign. If the MSE-gradient is positive implies the signal error increases positively, which means filter weights must decrease; and if the gradient is negative, the filter weights must increase in magnitude, [29]. There are cases the MSE-gradient value alternates in sign before attaining the steady-state; this is observed when the step size is 0.1 and 0.05 for LMS algorithm and 0.02 for NLMS algorithm. The study [43] describes this scenario as the algorithm is almost approaching the optimum solution and decelerating the gradient. MSE-gradient decelerates by decreasing individual step-size so that the steady state error will finally decrease. However, simple algorithms selecting individual step sizes for each filter tap based on the error estimation magnitude did not prove to be effective, [44], [45].

Visual assessments of the filter signal error convergence, filter weights MSE-gradient and filter output signal presented in Figure 5-8 accredited the initial filter step-size 0.0.2 to be close to best possible choice. Each figure presents LMS and NLMS algorithms visual performance based on three filter length values (16, 32 and 64) and same step-size $(\mu=0.02)$. Time domain simulation results present signal error convergences, filter weights MSE-gradient and filter output while filtered signal frequency spectrum is used to assess noise cancellation results. In subsection 4.2.1 and 4.2.2, MATLAB simulation learning curves are presented to substantiate the LMS and NLMS algorithm performances on non-stationary mud pulse signal application.

\subsubsection{LMS Algorithm Performance}

Simulation results show that, LMS algorithm converge slowly as the steady-state error falls slowly for each simulated filter length as shown in figure 5. As the filter length increases, the MSE-gradient value decreases positively, that implies, the error signal decreases positively. When the filter length is 16 , the steady state error is approximately 0.07843 , and when the filter length is 32 , the steady state error decreases to 0.04093 , subsequently when the filter length is increased to 64, the steady state error decreases further to 0.00006086 . 

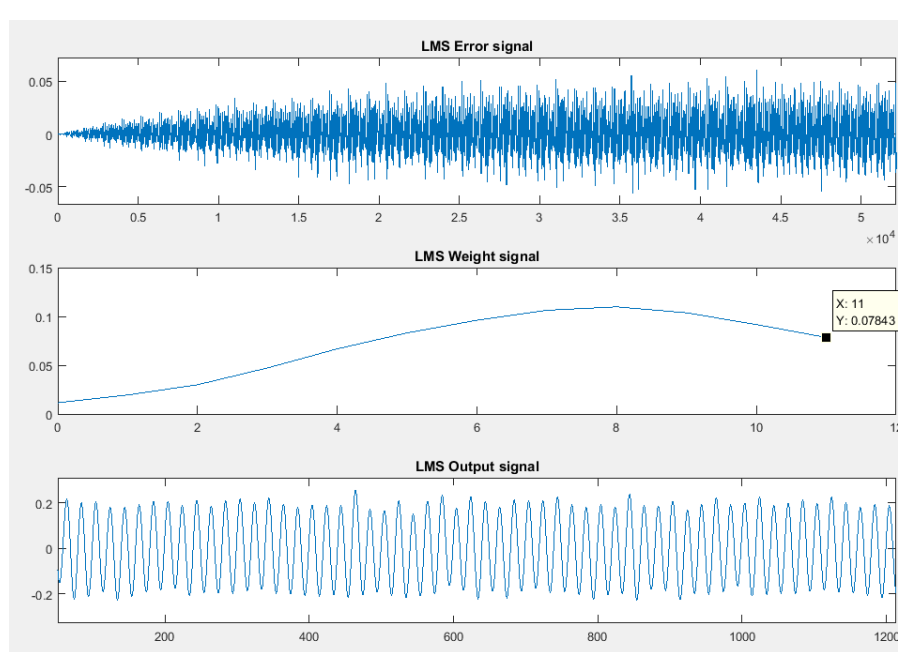

(a)

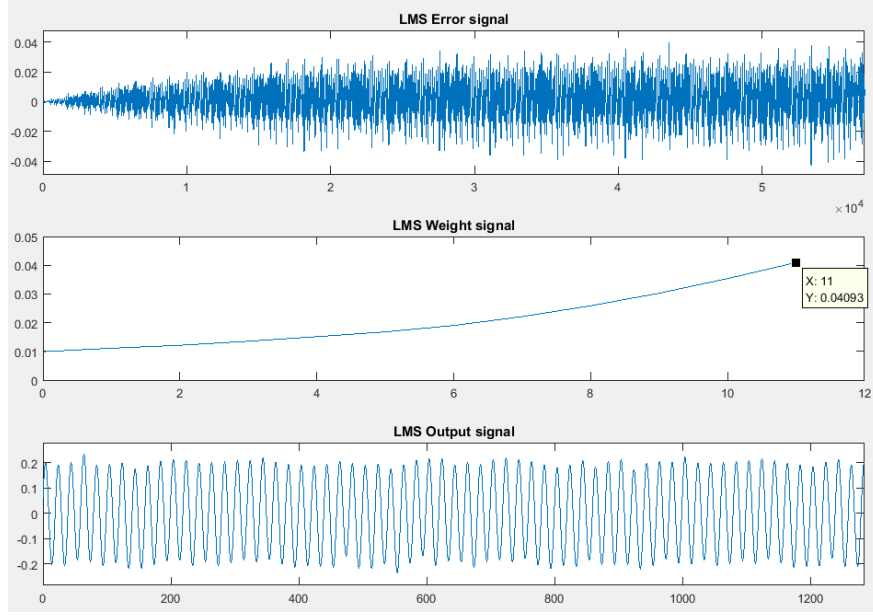

(b)

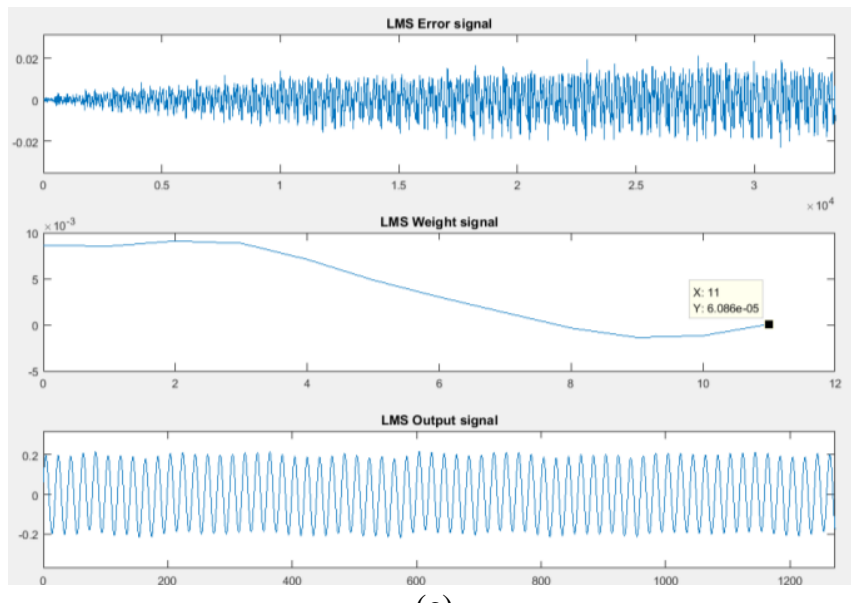

(c)

Figure 5: LMS Algorithm Filter Output- Time Domain: (a) Filter Length 16 Simulation, (b) Filter Length 32 LMS Simulation and (c) Filter Length 64 LMS Simulation.

LMS algorithm has stable steady-error rate and achieve good noise cancellation filter performance at higher filter lengths as presented in Figure 6. Frequency spectrum simulation waveform shows that when the filter length was set to 16 , the output signal doesn't approach the ideal input mud pulse signal, Figure 6(a). However, when the filter length value was set at 32 and 64, the LMS noise cancellation method generates good signal characteristics to match the input mud pulse signal, Figure 6(b) and 6(c). The filter frequency spectrum shows that the LMS algorithm can eliminate the noise in signal frequency band. Although there are certain noises residual in the extracted signal, the noise frequencies are outside the signal frequency band and can be eliminated using digital low-pass filter and improve the signal noise ratio (SNR).

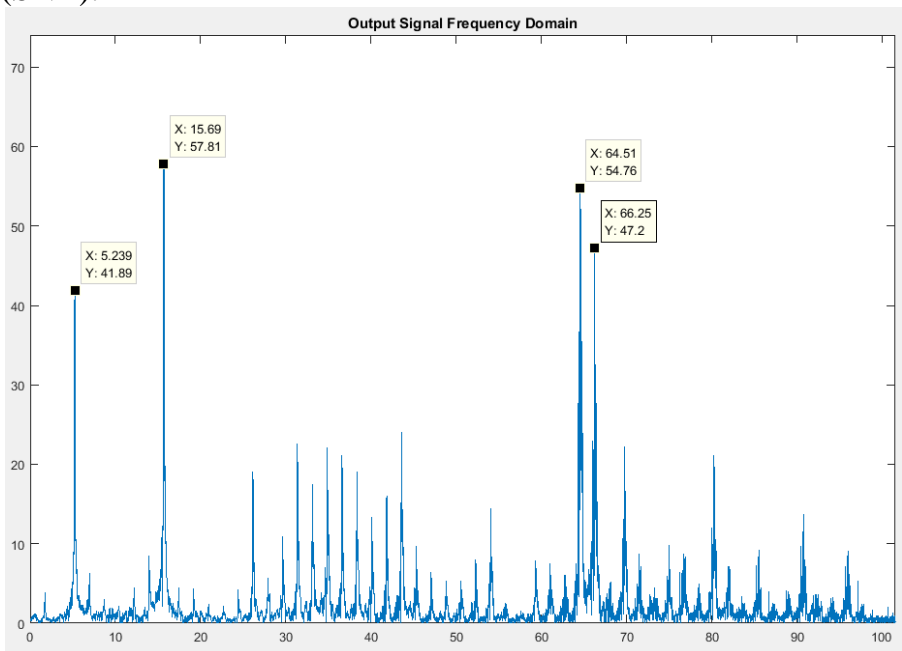

(a)

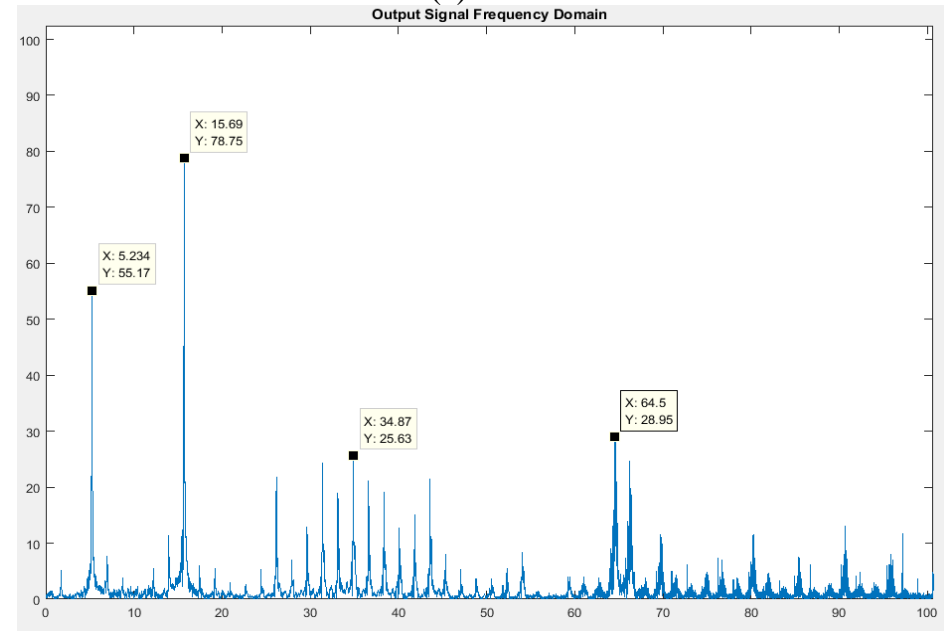

(b)

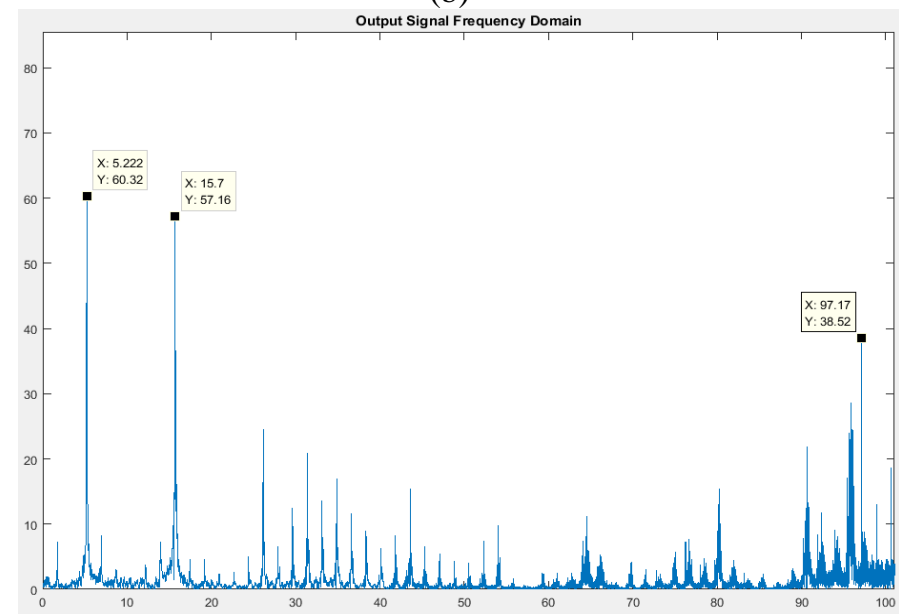

(c)

Figure 6: LMS Algorithm Filter Output- Frequency Spectrum: (a) Filter Length 16 Simulation, (b) (a) Filter Length 32 Simulation and (c) Filter Length 16 Simulation. 


\subsubsection{NLMS Algorithm Performance}

NLMS algorithm converges more quickly with fewer samples as the step size changes with time as shown in Figure 7. This algorithm uses adaptive step-size based on the input signal power to improve LMS convergence performance. The value of the MSE-gradient alternates in sign before attaining the steady-state. The algorithm is approaching the optimum solution and further improvement can be achieved by decreasing the individual step-size in order to decelerate MSE-gradient. However, the discrepancy between theoretical results based on this assumption decline the efficiency of this approach. NLMS algorithm becomes steady at lower values which is suitable for input signals that change slowly over time. When the filter length is 16 , the steady state error is approximately 0.006137 , Figure 7(a); increasing the filter length to 32 , the steady state error changes to 0.006458 , Figure 7(b); and when the filter length is 64 , the steady state error is approximately -0.02531 , Figure $7(\mathrm{c})$.
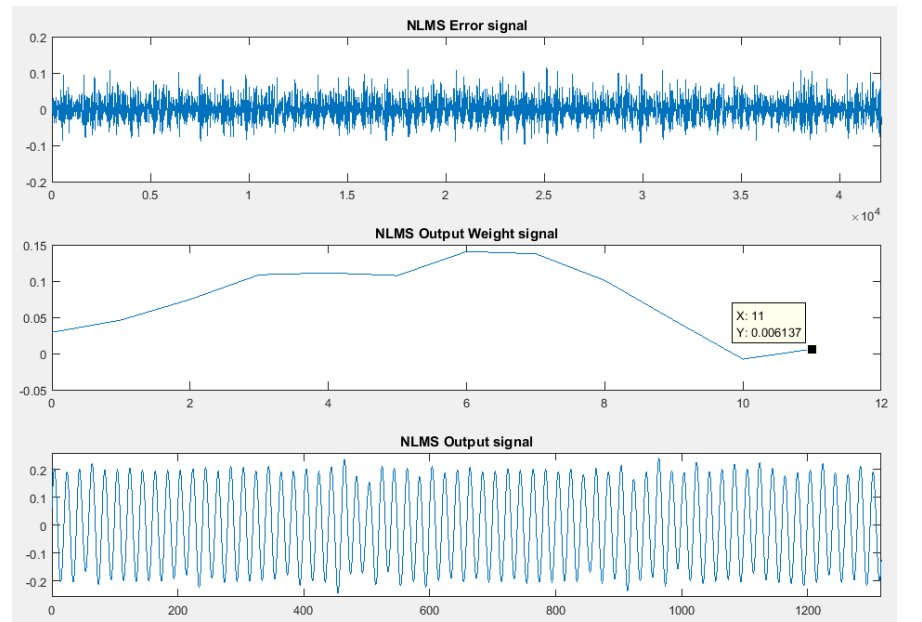

(a)

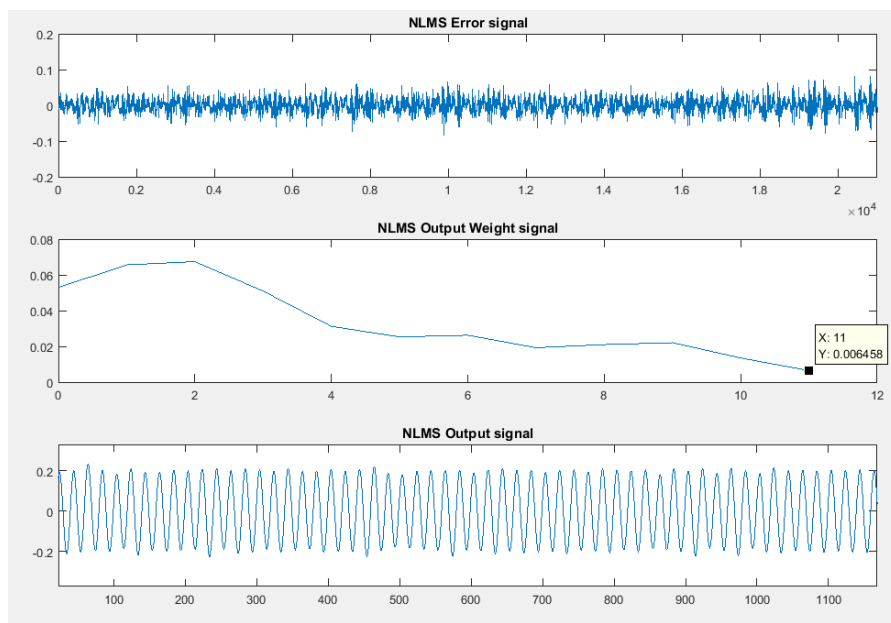

(b)

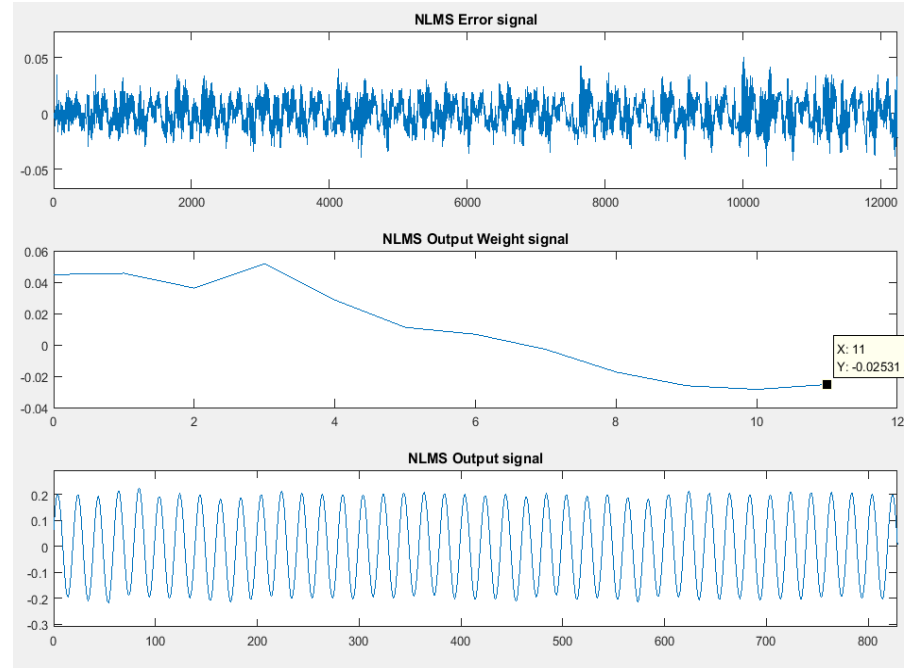

(c)

Figure 7: NLMS Algorithm Filter Output- Time Domain: (a) Filter Length 16 Simulation, (b) Filter Length 32 Simulation and (c) Filter Length 64 Simulation

NLMS algorithm has inefficiency noise cancellation performances at lower filter lengths, i.e. 16 and 32 values, as presented by frequency spectrum in Figure 8 . Extracted mud pulse signal contained visible noise harmonic components at lower frequencies, Figure 8(a) and 8(b). However, simulations result show noticeable noise cancellation improvement when the filter length is increased to 64 , Figure8(c). The presence of impulsive interference in either $d(n)$ or $u(n)$ degraded the NLMS algorithm performances.

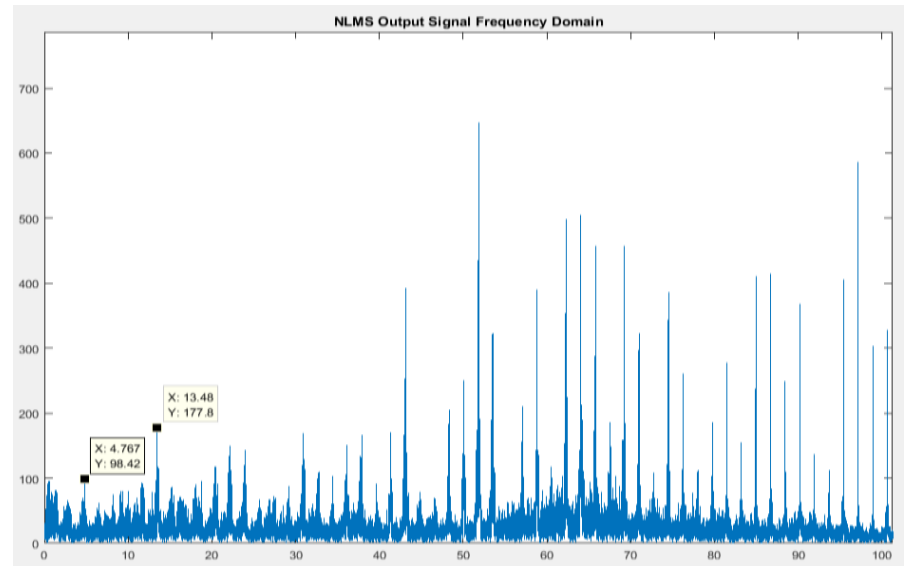

(a)

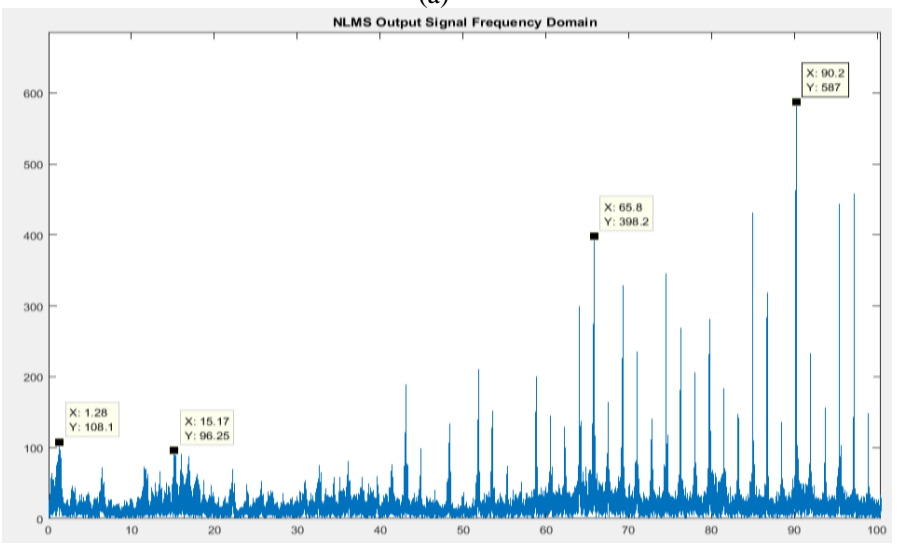

(b) 


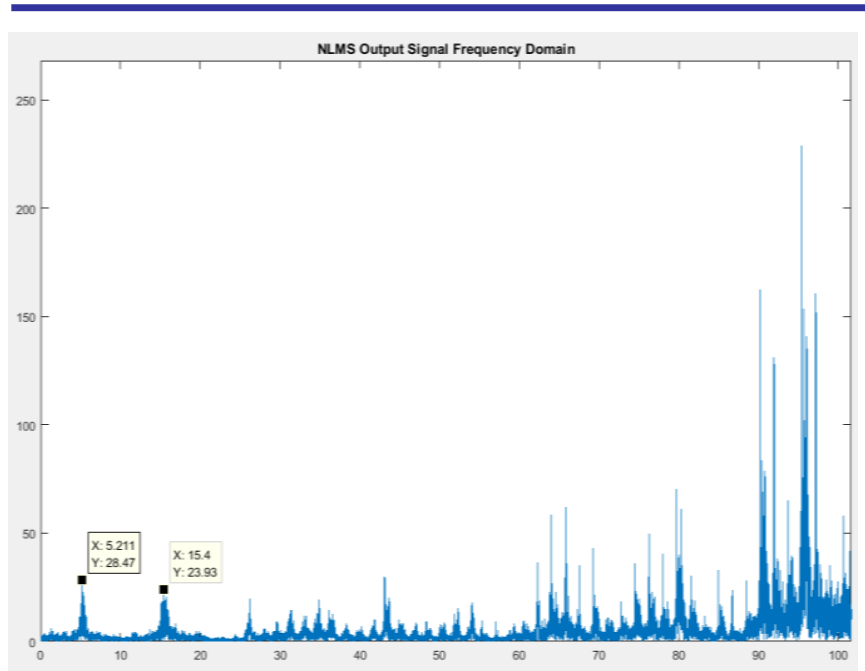

(c)

Figure 8: NLMS Algorithm Filter Output- Frequency Spectrum: (a) Filter Length 16 Simulation, (b) Filter Length 32 Simulation and (c) Filter Length 64 Simulation.

\subsection{CONCLUSION}

Adaptive LMS and NLMS algorithms are applied to eliminate mud pulse noises and interferential signals on field positive mud pulse signal. Initially, to avoid any undesirable adaptive simulation; the direct current (DC) frequency component was precisely estimated and removed from the mud pulse signal. To assess the signal extraction performances of the LMS and NLMS algorithms, MATLAB simulations are performed. The choices of the filter length and step-size parameters for both algorithms influence the quality of the extracted mud pulse signal. The step size is updated based on the input mud pulse signal and it controls the mean-square error (MSE) of the weighted-average gradient vector.

LMS algorithm has shown relatively good steady-state error stability and effective noise cancellation despite of slow convergence rate at the expense of computational time. LMS algorithm filtered signal frequency spectrum shows the method has good noise cancellation performance and can eliminate noise signals in the signal frequency band. Simulation results show certain noise frequency residuals in the extracted mud pulse signal; these noises are outside the signal frequency band. The residual noise frequencies can be eliminated using digital low-pass filter design and further improve signal noise ratio (SNR) quality. NLMS algorithm has faster convergence rate, rapid tracking, low misalignment and insufficient noise reduction capability at lower filter lengths; however noticeable noise cancellation improvements were observed at higher filter lengths. NLMS algorithm faster convergence rate is suitable for signal applications that change slowly over time.

An extensive set of simulation results across mud pulse signal demonstrated that the proposed approach can be applied into mud pulse LWD systems. LMS and NLMS algorithms showed improved practical noise cancellation application based on the signal and noise unknown statistical characteristics. This research presented step-by-step application of the method using MATLAB LMS variant methods and objects to yield satisfactory positive mud pulse noise cancellation simulation results.

\subsection{ACKNOWLEDGEMENT}

The authors wish to thank the anonymous reviewers for their valuable and constructive suggestions that improved this paper. The authors would like to declare no competing interests exist during the submission process of this manuscript and its publication approval. This research work was partly supported by the National Natural Science Foundation of China (nos. 51604038 and 51541408); the Laboratory for Marine Geology, Qingdao National Laboratory for Marine Science and Technology (no. MGQNLMKF201705) and Wenhai Program of QNLM (no. 2017WHZZB0401); the Research Fund for the Taishan Scholar Project of Shandong Province (no. TSPD20161007); and the Department of Education, Hubei Province (no. D20141303).

\subsection{REFERENCES}

[1] R. Hutin, R. W. Tennent, and S. V. Kashikar, 'New mud pulse telemetry techniques for deepwater applications and improved realtime data capabilities', in SPE/IADC drilling conference, 2001.

[2] C. Klotz, P. R. Bond, I. Wassermann, and S. Priegnitz, 'A new mud pulse telemetry system for enhanced MWD/LWD applications', in IADC/SPE Drilling Conference, 2008.

[3] C. Klotz, A. Kaniappan, A. K. Thorsen, E. Nathan, M. Jahangir, and L. Lie, 'A new mud pulse telemetry system reduces risks when drilling complex extended reach wells', in IADC/SPE Asia Pacific Drilling Technology Conference and Exhibition, 2008.

[4] C. Klotz, I. Wassermann, and D. Hahn, 'Highly flexible mud-pulse telemetry: a new system', in SPE Indian Oil and Gas Technical Conference and Exhibition, 2008.

[5] E. S. Shearer, Pulse signaling for downhole telemetry. US 2010/0188253 A1, 2010.

[6] B. Tu, E. H. Lin, and M. M. Ji, 'Research on mud pulse signal data processing in MWD', EURASIP Journal on Advances in Signal Processing, vol. 2012, no. 1, p. 182, 2012.

[7] J. Zhao, L. Wang, L. Fan, and Y. Liu, 'An effective approach for the noise removal of mud pulse telemetry system', in Electronic Measurement and Instruments, 2007. ICEMI'07. 8th International Conference on, 2007, pp. 1-971.

[8] F. Yuan and X. Gong, 'Research on signal processing of continuous wave mud pulse', in Mechanic Automation and Control Engineering (MACE), 2011 Second International Conference on, 2011, pp. 627631.

[9] Q. Zhao, B. Zhang, and H. Hu, 'Novel two-step filtering scheme for a logging-while-drilling system', Computer Physics Communications, vol. 180, no. 9, pp. 1566-1571, 2009.

[10] H.-U. Brackel, Pump noise reduction and cancellation. US20140017092, 2016.

[11] A. G. Brooks, Mud pulse telemetry noise reduction method. US 9,007,232 B2, 2015.

[12] F. Qu, Z. Zhang, J. Hu, J. Xu, S. Wang, and Y. Wu, 'Adaptive dualsensor noise cancellation method for continuous wave mud pulse telemetry', Journal of Petroleum Science and Engineering, 2017.

[13] H. Reckmann, Downhole noise cancellation in mud-pulse telemetry. US 2008/0074948 A1, 2008.

[14] N. Priyadarshani, S. Marsland, I. Castro, and A. Punchihewa, 'Birdsong denoising using wavelets', PloS one, vol. 11, no. 1, p. e0146790, 2016.

[15] D. Yuan, X. Cui, G. Wang, J. Jin, D. Fan, and X. Jia, 'Study on the GPS Data De-noising Method Based on Wavelet Analysis', in International Conference on Computer and Computing Technologies in Agriculture, 2011, pp. 390-399.

[16] L. Guan and Y. Du, 'Review of the Application of Wavelet Analysis in the Petroleum Industry', in Canadian International Petroleum Conference, 2004.

[17] C.-W. Li, D.-J. Mu, A.-Z. Li, Q.-M. Liao, and J.-H. Qu, 'Drilling mud signal processing based on wavelet', in Wavelet Analysis and 
Pattern Recognition, 2007. ICWAPR'07. International Conference on, 2007, vol. 4, pp. 1545-1549.

[18] M. A. Namuq, M. Reich, and S. Bernstein, 'Continuous wavelet transformation: A novel approach for better detection of mud pulses', Journal of Petroleum Science and Engineering, vol. 110, pp. 232242, 2013.

[19] W.-Y. Chen, B. Fang, and Y. Wang, 'MWD drilling mud signal Denoising and signal extraction research based on the pulse-code information', in Wavelet Analysis and Pattern Recognition (ICWAPR), 2010 International Conference on, 2010, pp. 244-249.

[20] J. Li and H. Reckmann, 'System and method for pump noise cancellation in mud pulse telemetry', 7577528, Aug. 2009.

[21] K. Liu, 'Adaptive Noise Cancellation for Electromagnetic-whileDrilling System', in Information Science and Control Engineering (ICISCE), 2016 3rd International Conference on, 2016, pp. 1253 1256.

[22] Y. Shen, L. Zhang, H. Zhang, Y. Su, L. Sheng, and L. Li, 'Eliminating Noise of Mud Pressure Phase Shift Keying Signals with A Self-Adaptive Filter', Indonesian Journal of Electrical Engineering and Computer Science, vol. 11, no. 6, pp. 3028-3035, 2013.

[23] Y. Shen, L.-T. Zhang, S.-L. Cui, L.-M. Sheng, L. Li, and Y.-N. Su, 'Delay pressure detection method to eliminate pump pressure interference on the downhole mud pressure signals', Mathematical Problems in Engineering, vol. 2013, 2013.

[24] J. Shao et al., 'Differential signal extraction for continuous wave mud pulse telemetry', Journal of Petroleum Science and Engineering, vol. 148, pp. 127-130, 2017.

[25] S. S. Haykin, Adaptive filter theory, 3rd ed. Pearson Education India, 2008.

[26] K. R. Borisagar and G. R. Kulkarni, 'Simulation and comparative analysis of LMS and RLS algorithms using real time speech input signal', Global Journal of Research In Engineering, vol. 10, no. 5, 2010.

[27] K. R. Borisagar and G. R. Kulkarni, 'Simulation and performance analysis of adaptive filter in real time noise over conventional fixed filter', in Communication Systems and Network Technologies (CSNT), 2012 International Conference on, 2012, pp. 621-624.

[28] Y. He, H. He, L. Li, Y. Wu, and H. Pan, 'The applications and simulation of adaptive filter in noise canceling', in Computer Science and Software Engineering, 2008 International Conference on, 2008 , vol. 4, pp. 1-4.

[29] J. Dhiman, S. Ahmad, and K. Gulia, 'Comparison between Adaptive filter Algorithms (LMS, NLMS and RLS)', International Journal of Science, Engineering and Technology Research (IJSETR), vol. 2, no. 5, pp. 1100-1103, 2013.

[30] A. Pandey, L. D. Malviya, and V. Sharma, 'Comparative study of LMS and NLMS algorithms in adaptive equalizer', International Journal of Engineering Research and Applications (IJERA), vol. 2, no. 3, pp. 1584-1587, 2012.

[31] E. Soria, J. Calpe, J. Chambers, M. Martínez, G. Camps, and J. D. M. Guerrero, 'A novel approach to introducing adaptive filters based on the LMS algorithm and its variants', IEEE transactions on education, vol. 47, no. 1, pp. 127-133, 2004

[32] J. Yadav, M. Kumar, R. Saxena, and A. K. Jaiswal, 'performance analyis of LMS adaptive FIR filter and RLS adaptive fir filter for noise cancellation', Signal \& Image Processing, vol. 4, no. 3, p. 45 , 2013.

[33] R. K. Thenua and S. K. Agarwal, 'Simulation and performance analysis of adaptive filter in noise cancellation', International Journal of Engineering Science and Technology, vol. 2, no. 9, pp. 4373-4378, 2010.

[34] K. Sahu and R. Sinha, 'Simulation of NLMS Adaptive Filter for Noise Cancellation', Engineering and Applied Sciences, vol. Volume2, no. Issue-1, pp. 27-29, Jan. 2015.

[35] H.-C. Huang and J. Lee, 'A new variable step-size NLMS algorithm and its performance analysis', IEEE Transactions on Signal Processing, vol. 60, no. 4, pp. 2055-2060, 2012.

[36] M. Li and X. Xi, 'A new variable step-size nlms adaptive filtering algorithm', in Information Technology and Applications (ITA), 2013 International Conference on, 2013, pp. 236-239.

[37] H. Takekawa, T. Shimamura, and S. Jimaa, 'An efficient and effective variable step size NLMS algorithm', in Signals, Systems and Computers, 2008 42nd Asilomar Conference on, 2008, pp. 16401643.
[38] A. Carusone and D. A. Johns, 'Obtaining digital gradient signals for analog adaptive filters', in Circuits and Systems, 1999. ISCAS'99. Proceedings of the 1999 IEEE International Symposium on, 1999, vol. 3, pp. 54-57.

[39] D. Mali, 'Comparison of DC Offset Effects on LMS Algorithm and its Derivatives', International Journal of Recent Trends in Engineering, vol. 1, no. 1, p. 323, 2009.

[40] A. C. Carusone, Digital algorithms for analog adaptive filters. PhD Thesis, University of Toronto, 2002.

[41] D. Bismor, K. Czyz, and Z. Ogonowski, 'Review and comparison of variable step-size LMS algorithms', International Journal of Acoustics and Vibration, vol. 21, no. 1, pp. 24-39, 2016

[42] J.-K. Hwang and Y.-P. Li, 'Variable step-size LMS algorithm with gradient-based weighted average', IEEE Signal Processing Letters, vol. 16, no. 12, pp. 1043-1046, 2009.

[43] D. Bismor, 'LMS algorithm step size adjustment for fas convergence', Archives of Acoustics, vol. 37, no. 1, pp. 31-40, 2012.

[44] W.-P. Ang and B. Farhang-Boroujeny, 'A new class of gradien adaptive step-size LMS algorithms', IEEE transactions on signal processing, vol. 49, no. 4, pp. 805-810, 2001.

[45] V. J. Mathews and Z. Xie, 'A stochastic gradient adaptive filter with gradient adaptive step size', IEEE Transactions on Signal Processing, vol. 41, no. 6, pp. 2075-2087, 1993. 